\title{
Berberine Attenuates Arterial Plaque Formation in Atherosclerotic Rats with Damp-Heat Syndrome via Regulating Autophagy
}

This article was published in the following Dove Press journal:

Drug Design, Development and Therapy

\begin{abstract}
Xiao Ke, ${ }^{1,2, *}$ Yiteng Huang, ${ }^{2, *}$ Liang Li, ${ }^{3}$ Fuya Xin, ${ }^{3}$ Luhua $\mathrm{Xu}{ }^{3}$ Yuangui Zhang, ${ }^{3}$

Zhicong Zeng, ${ }^{3}$ Fengxia Lin, (D) ${ }^{3}$ Yinzhi Song ${ }^{3}$

'Key Laboratory of Biomaterials of Guangdong Higher Education Institutes, Guangdong Provincial Engineering and Technological Research Center for Drug Carrier Development, Department of Biomedical Engineering, Jinan University, Guangzhou, Guangdong 510632, People's Republic of China; ${ }^{2}$ Department of Cardiology, Fuwai Hospital, Chinese Academy of Medical Sciences, Shenzhen, (Shenzhen Sun Yat-Sen Cardiovascular Hospital), Shenzhen, Guangdong 518057 , People's Republic of China; ${ }^{3}$ Department of Cardiology, Shenzhen Bao'an Traditional Chinese Medicine Hospital Group, The Affiliated Hospital of Guangzhou University of Chinese Medicine, Shenzhen 518133, People's Republic of China
\end{abstract}

*These authors contributed equally to this work

Correspondence: Fengxia Lin Department of Cardiology,Shenzhen Bao'an Traditional Chinese Medicine Hospital Group, The Affiliated Hospital of Guangzhou University of Chinese

Medicine, Shenzhen 518133, People's

Republic of China

Tel +8617301933930

Email szlinfx@163.com

Yinzhi Song

Department of Cardiology, Shenzhen Bao'an Traditional Chinese Medicine Hospital Group, The Affiliated Hospital of Guangzhou University of Chinese

Medicine, Shenzhen 518133, People's

Republic of China

Tel +86I3760298578

Email songyinzhi@126.com
Purpose: Berberine (BBR) is an effective component of Huanglian and has shown to attenuate atherosclerosis (AS); however, the detailed mechanism of BBR-mediated protective actions against AS remains elusive. This study was undertaken to examine the effects of BBR on aortic atherosclerotic plaque stability and the expression of autophagy-related proteins in AS rats with damp-heat syndrome or yang deficiency.

Methods: Thirty SD rats were randomly divided into (1) control (CON); (2) damp-heat syndrome atherosclerosis (AS + DH); (3) yang deficiency syndrome atherosclerosis (AS + YX); (4) dampheat syndrome atherosclerosis + BBR (AS + DH + BBR); (5) yang deficiency syndrome, atherosclerosis + BBR (AS + YX + BBR); and (6) damp-heat syndrome, atherosclerosis + BBR +3 -methyladenine $(\mathrm{AS}+\mathrm{DH}+\mathrm{BBR}+3-\mathrm{MA})(\mathrm{n}=5$ /group $)$ groups. Pathological morphology, macrophage plaque infiltration, inflammation, and LC3-II and P62 expression were assessed.

Results: Compared with the CON group, the AS + DH and AS + YX groups had an increased plaque area in the aortic tissue with substantial foam cell and macrophage infiltration, and increased levels of IL-1 $\beta$ and TNF- $\alpha(\mathrm{P}<0.01)$. After four weeks of BBR intervention, the plaque area in the $\mathrm{AS}+\mathrm{DH}+\mathrm{BBR}$ group was reduced with decreased foam cells and macrophage infiltration, and decreased levels of TNF- $\alpha$ and IL-1 $\beta$, whereas LC3-II protein expression was increased and P62 protein expression was decreased in the AS $+\mathrm{DH}+\mathrm{BBR}$ group when compared to $\mathrm{AS}+\mathrm{DH}$ group. In addition, the $\mathrm{AS}+\mathrm{DH}+\mathrm{BBR}+$ 3-MA group exhibited a significantly enlarged plaque, substantial foam cell and macrophage infiltration, increased levels of IL-1 $\beta$ and TNF- $\alpha$, and decreased LC3-II and P62 $(\mathrm{P}<0.01)$ expression when compared to the $\mathrm{AS}+\mathrm{DH}+\mathrm{BBR}$ group.

Conclusion: Our results indicated that the BBR could inhibit arterial plaque formation and alleviate the inflammatory response in the aortic tissues in the AS rats with damp-heat syndrome possibly via promoting autophagy. The molecular mechanisms of BBR-mediated protective effects in this animal model still require further investigation.

Keywords: berberine, autophagy, LC3-II, P62, arterial plaque, damp-heat syndrome

\section{Introduction}

Atherosclerosis (AS) as a common pathological basis of cardiovascular and cerebrovascular diseases is accelerated by ongoing inflammatory and lipid metabolism disorders. According to epidemiological data, the mortality rate of AS-related vascular diseases in individuals aged older than 35 years has decreased from $16 \%$ in 1980 to $4 \%$ in $2010 ;^{1}$ however, the incidence of AS in China remains high with one-third of people in China suffering from carotid atherosclerosis. ${ }^{2}$ Thus, further understanding of the pathogenesis of AS and the proposal of more effective 
prevention and treatment measures are the urgent issues to be solved in clinical practice. Autophagy is a process by which organelles and the cellular contents are degraded by the lysosomal system, which is an important means of maintaining the stability of the intracellular environment and achieving self-renewal of the organelles. ${ }^{3}$ Autophagy plays an important role in the stabilization of AS plaques. Recent studies have found that autophagy involves in the inflammation and oxidative stress in AS plaque cells, and targeting the autophagy-related markers may provide a new therapeutic strategy in alleviating the development and progression of AS. ${ }^{4}$

Coptidis rhizoma (also known as Huanglian in Chinese) is a traditional Chinese medicine with the effects of resolving dampness, clearing away heat, detoxification, and purging fire. ${ }^{5}$ Berberine (BBR) is the one of major active components in coptidis rhizoma and it has various biological functions including anti-inflammatory, lipidlowering, anti-platelet and anti-oxidative functions. ${ }^{6,7}$ As a representative of heat-clearing and moisturizing drugs, BBR appears in a large number of classic prescriptions for clearing heat and dampness. Both clinical and experimental studies have found that BBR was effective in attenuating the development and progression of AS. ${ }^{8,9}$ Studies also demonstrated that anti-atherosclerotic effect of BBR may be partly due to changes in composition and functions of gut microbiota. ${ }^{10}$ Recently, there is growing evidence showing that exerted the protective effects in cardiovascular diseases via modulating autophagy. ${ }^{11}$ Huang et al showed that BBR alleviated cardiac ischemia/reperfusion injury by suppressing excessive autophagy in cardiomyocytes; ${ }^{12} \mathrm{Li}$ et al demonstrated BBR improved pressure-induced cardiac hypertrophy and dysfunction via inducing autophagy. ${ }^{13}$ In AS studies, Fan et al demonstrated that BBR alleviated ox-LDL induced inflammatory factors in AS via up-regulation of autophagy; ${ }^{14}$ while the exact mechanisms of BBR-mediated autophagy in AS are largely unknown.

According to the TCM theory, the treatment is based on the holistic concept and the theory of syndrome differentiation. According to the theory of TCM, the causative agent/ factor of diseases can be summarized as heat, damp, cold and toxin. ${ }^{15}$ The damp-heat syndrome is an acute exogenous febrile disease caused by pathogenic dampness, and the causes of damp-heat syndrome are multifactorial including not only environmental factors, but also autoimmune function and pathogenic microorganism. ${ }^{16}$ Studies found that Baicalin is curative against rotavirus damp heat diarrhea by tuning colonic mucosal barrier and lung immune function ${ }^{17}$ high dose of Haoqinqingdan decoction can improve the damp-heat syndrome in rats with influenza viral pneumonia. ${ }^{16}$ Yin-yang theory is an essential part of TMC in diagnosis and treatment based on overall analysis of symptoms and phenotypes fo the body. Yang-deficiency is associated with the yin-yang imbalance points at the functional lowlife showing various symptoms such as lassitude and impotence, cold-blooded and cold feared, lumbago and fatigue. ${ }^{18}$ Studies found that immunotoxicity of hydrocortisone on Th1/Th2-related cytokine production Is associated with yang-deficient state in TCM. ${ }^{18}$ Er Shen Wan extract alleviated polyuria in a rat model of spleen-kidney Yang deficiency-induced diarrhea. ${ }^{19}$ In addition, Integrated adrenal and testicular metabolomics revealed the protective effects of Guilingji on the Yang deficiency syndrome rats. ${ }^{20}$

In this study, we established an AS rat model with the characteristics of damp-heat syndrome or yang deficiency syndrome and examined the pathological morphology and degree of the inflammatory response in aortic tissue sections following BBR intervention. Further mechanistic studies were also performed to explore the effects of BBR on the expression of autophagy-related proteins in AS rats with damp-heat syndrome or yang deficiency. The present study will provide novel insights into the protective effects of BBR in AS.

\section{Materials and Methods Experimental Animals and Ethics Statement}

A total of 30 specific pathogen-free (SPF) Sprague Dawley SD male rats (16 weeks old, 180-200 g) were purchased from the Experimental Animal Center of Guangzhou University of Traditional Chinese Medicine (license number: SCXK [Guangdong] 2016-0019). The experiment was approved by the ethics committee of Shenzhen Bao 'an Hospital of Traditional Chinese Medicine (group), and implemented in strict accordance with the National Guidelines for Experimental Animal Welfare (the Ministry of Science and Technology, China).

\section{Animal Models and Drug Treatments}

According to the method reported in the study, ${ }^{16,21}$ The rats were housed in an SPF environment under a light/ dark time of $12 \mathrm{~h} / 12 \mathrm{~h}$. The normal group was fed a common diet $(23 \%$ flour, $20 \%$ bran, $22 \%$ soybean meal, $20 \%$ corn, $10 \%$ rice meal, $2.5 \%$ bone meal, and $2.5 \%$ fish meal) and housed in the environment under an 
ambient temperature of $25 \pm 1{ }^{\circ} \mathrm{C}$ with a relative humidity of $40 \%-50 \%$; the model group was fed a high-fat and high-sugar diet $(83.25 \%$ common diet, $1.5 \%$ cholesterol, $0.25 \%$ sodium cholate, $10 \%$ lard, and $5 \%$ sucrose) for 12 weeks. For the AS rats with damp-heat syndrome, the AS rats were housed in the environment under an ambient temperature of $32 \pm 1{ }^{\circ} \mathrm{C}$, at a relative humidity of $65-70 \%$ according to previous studies. ${ }^{16,22}$ For the AS rats yang deficiency, rats were placed in a refrigerator at $2^{\circ} \mathrm{C}$ for $2 \mathrm{~h}$ every day according to the previous studies. $^{20,23}$

A total of $30 \mathrm{SD}$ rats were randomly divided into a normal group (CON), AS with damp-heat syndrome group (AS + DH), AS with yang deficiency group (AS + YX), AS with damp-heat syndrome treated with BBR (Shanghai Sangon Biological Co., Ltd.; Shanghai, China) group (AS + DH + BBR), AS with yang deficiency syndrome treated with BBR (AS + YX + BBR), AS with damp-heat syndrome treated with BBR and 3-methyl adenine (3-MA; an autophagy inhibitor; Lanzhou Day State Biological Technology Co., Ltd., Lanzhou, China) group (AS + DH + BBR + 3-MA) group. For the AS + $\mathrm{DH}+\mathrm{BBR}$ and $\mathrm{AS}+\mathrm{YX}+\mathrm{BBR}$ groups, rats were orally administered BBR $(150 \mathrm{mg} / \mathrm{kg})$ daily for four weeks. The dose standard was based on previous studies to have good safety. ${ }^{24}$ For the AS + DH + BBR + 3-MA group, rats were orally administered BBR $(150 \mathrm{mg} / \mathrm{kg})$ daily and 3-MA $(100 \mathrm{mg} / \mathrm{kg}$, intraperitoneally) every two days for four weeks.

\section{Hematoxylin and Eosin (HE) Staining}

The ascending aortic tissue sections were deparaffinized with xylene for 5-10 min, stained with hematoxylin for $5 \mathrm{~min}$, and then separated with a $1 \%$ hydrochloric acid alcohol in a saturated solution of lithium carbonate. After a short period of bluening, the sections were quickly washed in distilled water, stained with approximately $0.1-0.5 \%$ eosin dye solution for 1-5 min, and finally dehydrated with gradient alcohol. The degree of aortic pathology was observed with an optical microscope (200×) and the samples were photographed. The sections were analyzed using Image-Pro Plus 6.0 image processing software.

\section{Immunofluorescence Staining}

After the samples were dewaxed with xylene and hydrated with alcohol, they were washed in phosphate buffered saline (PBS), and the tissue antigen retrieval was prepared in citrate buffer. Then, $50 \mu \mathrm{L}$ of $3 \% \mathrm{H}_{2} \mathrm{O}_{2}$ was added dropwise to the section for $10 \mathrm{~min}$ at room temperature, and PBS was used to wash the samples before blocking with $5 \%$ bovine serum albumin (BSA). After that, the sections were incubated with the corresponding primary antibody overnight at $4^{\circ} \mathrm{C}$ followed by incubating a fluorescent secondary antibody. Finally, the sections were stained with $0.5 \mu \mathrm{g} / \mathrm{mL}$ DAPI $10 \mathrm{~min}$ for counterstaining. The samples were observed and photographed under a fully automatic fluorescence microscope $(400 \times)$.

\section{Detection of Changes in Interleukin (IL)- I $\beta$ and Tumor Necrosis Factor (TNF)- $\alpha$ Expression by Enzyme-Linked Immunosorbent Assay (ELISA)}

Blood samples were collected from the tail vein before the rats were sacrificed, anticoagulated with 5\% EDTA, centrifuged at $800 \mathrm{xg}$, and the plasma was separated and stored at $-20^{\circ} \mathrm{C}$ for further examination. The level of IL-1 $\beta$ and TNF- $\alpha$ expression was measured by the corresponding ELISA kits according to the manufacturer's instructions.

\section{Immunohistochemical Detection of LC3-II and P62 Expression}

The samples were deparaffinized with xylene for 5-10 min and re-hydrated in alcohol. Samples were washed in phosphate buffered saline (PBS), and the tissue antigen retrieval was prepared in citrate buffer. Then, the samples were incubated with $3 \% \mathrm{H}_{2} \mathrm{O}_{2}$ for 5-10 min at room temperature, and PBS was used to wash the samples before blocking with 5\% BSA. After that, the sections were incubated with the corresponding primary antibody overnight at $4^{\circ} \mathrm{C}$ followed by incubating with the corresponding horseradish peroxidaseconjugated secondary antibodies for $1 \mathrm{~h}$ at room temperature. The stained proteins were visualized using the 3, 3-diaminobenzidine chromogen. An optical microscope $(200 \times)$ was used to observe the samples and take photos, and Image-pro Plus 6.0 Image processing software was used to analyze the images.

\section{Western Blot Analysis of LC3-II and P62 Protein Expression}

Protein samples were extracted from rat aortic tissues by using RIPA buffer (Sigma-Aldrich, St. Louis, USA), and the protein concentrations were measured by a BCA kit (Blue Skies in Shanghai Biological Technology Co., Ltd; Shanghai, China) according to the manufacturer's protocol. A total of $50 \mu \mathrm{g}$ protein samples were resolved on a $10 \%$ SDS-PAGE followed by transferring the proteins to 
a PVDF membrane via the semi-dry transfer method. After incubating with $1.5 \%$ milk at room temperature for $1 \mathrm{~h}$, the membranes were incubated with corresponding primary antibodies including LC3-II (dilution ratio 1:3000; Cell Signaling Technology, Danvers, USA), P62 (dilution ratio 1:1000; Cell Signaling Technology) and GAPDH (1:3000; Cell Signaling Technology) overnight at $4^{\circ} \mathrm{C}$. After that, the membranes were incubated corresponding secondary antibody for $2 \mathrm{~h}$ at room temperature. The Western blot bands were visualized by using the ECL kit according to the manufacturer's protocol. The relative protein expression levels were analyzed using the Biosens gel image processing system and GAPDH was used as the internal control.

\section{Statistical Analysis}

All the statistical analysis was performed by using the GraphPad software (Version 6.0, La Jolla, USA). Data were presented as mean \pm standard deviation. Significant difference among treatment groups was evaluated by oneway ANOVA followed by Bonferroni's multiple comparison test. $\mathrm{P}<0.05$ was considered statistically significant.

\section{Results}

\section{Symptoms and Signs Changes of Each} Group and Pathological Changes in the Aortic Tissues

After modeling, rats in the AS + DH group, $\mathrm{AS}+\mathrm{DH}+\mathrm{BBR}$ group, and AS + DH + BBR + 3-MA group showed symptoms of lack of energy, reduced activity, sparse and dull hair accompanied with frequent diarrhea. Rats in the AS + YX group and AS + YX + BBR group showed chills, flock together, arched backs, less activity, dry hair, loss of appetite, burnout, weight loss accompanied with diarrhea.

As shown in Figure 1A and B, the AS + DH and AS + YX group showed irregular intima morphology and significant intima hyperplasia, with differential nuclear morphology and size, as well as disordered arrangement. A large amount of lipid plaque deposition and foam cell formation was observed in the intima, and the percentage of the plaque area was significantly increased when compared to the CON group $(\mathrm{P}<0.01)$. In the AS + DH + BBR group, BBR intervention was associated with a thinning of the intima, uniform nuclear morphology, significant reduction in lipid plaques and foam cells, and the plaque area ratio was significantly reduced when compared to AS + DH group $(\mathrm{P}<0.01)$; however, there was no significant change in the aortic histopathological morphology between the AS + YX + BBR group and AS + YX group. Further analysis revealed that lipid plaques and foam cells were significantly increased in the AS $+\mathrm{DH}+\mathrm{BBR}$ + 3-MA group with thickened and irregular intima, variable nuclear shapes and sizes, as well as a significant increase in disordered arrangement and plaque area ratio when compared to $\mathrm{AS}+\mathrm{DH}+\mathrm{BBR}$ group $(\mathrm{P}<0.01)$.

\section{Changes of Macrophage Infiltration in the Aortic Tissues}

The changes in macrophage infiltration in the aortic tissues are shown in Figure 2. The AS + DH and the AS + YX groups displayed a large amount of macrophage infiltration in the aortic tissue sections, and the rate of CD68 positive expression was significantly increased when compared to CON group $(\mathrm{P}<0.01)$. In the $\mathrm{AS}+\mathrm{DH}+\mathrm{BBR}$ group, BBR intervention decreased the number of macrophages and expression of CD86 when compared to AS + DH group $(\mathrm{P}<0.01)$, whereas there was no significant difference between $\mathrm{AS}+\mathrm{YX}+\mathrm{BBR}$ and $\mathrm{AS}+\mathrm{YX}$ groups $(\mathrm{P}>0.05)$. In contrast, compared with the AS + DH + BBR group, there was a substantial number of infiltrating macrophages and the level of positive CD68 expression was significantly increased in AS + DH + BBR + 3-MA group $(\mathrm{P}<0.01)$.

\section{Changes of the Expression of Inflammatory Factors in the Aortic Tissues}

As shown in Figure 3, changes in the level of IL- $1 \beta$ and TNF- $\alpha$ expression were detected by the ELISA kits. The expression levels of inflammatory factors (IL- $1 \beta$ and TNF- $\alpha$ ) in the AS + $\mathrm{DH}$ group and $\mathrm{AS}+\mathrm{YX}$ group were significantly increased when compared to the CON group ( $\mathrm{P}<0.01)$. After treatment with BBR, the levels of IL- $1 \beta$ and TNF- $\alpha$ in the AS + DH + BBR group were significantly decreased when compared to the AS + DH group $(\mathrm{P}<0.01)$, BBR treatment failed to attenuate the increase in these inflammatory factors in the $\mathrm{AS}+\mathrm{YX}$ rats $(\mathrm{P}>0.05)$. Moreover, the levels of IL-1 $\beta$ and TNF- $\alpha$ expression were significantly increased in the AS + $\mathrm{DH}+\mathrm{BBR}+3$-MA group compared with that of the AS + DH + BBR group $(\mathrm{P}<0.01)$.

\section{Changes of LC3-II and P62 Protein Expression Levels in the Aortic Tissues as Determined by Immunostaining}

The protein expression LC3-II and P62 as determined by immunostaining in the aortic tissue are presented in Figures 4 and 5. There was no significant difference in the 
A
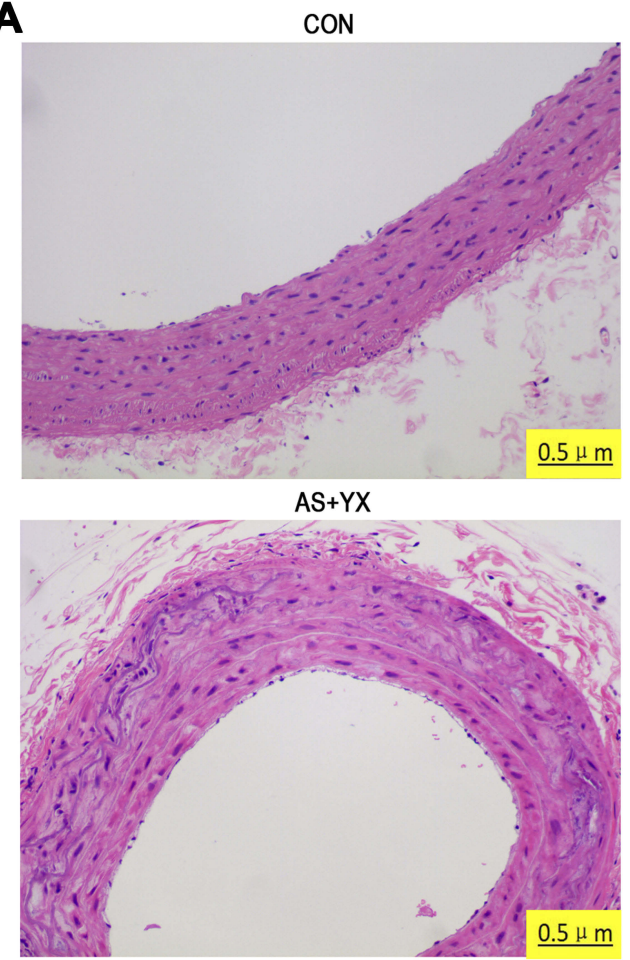

$A S+Y X+B B R$

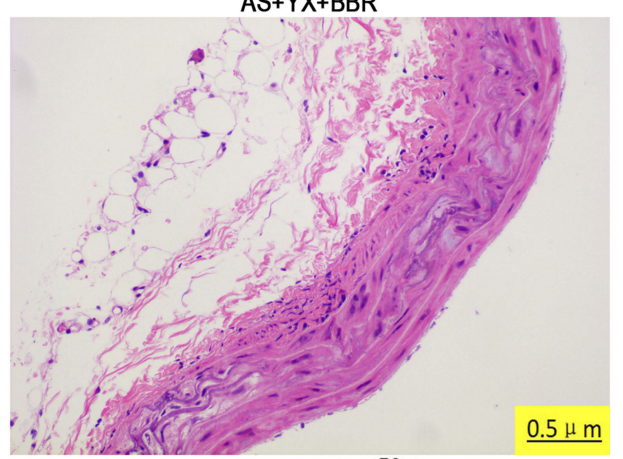

$\mathrm{AS}+\mathrm{DH}$

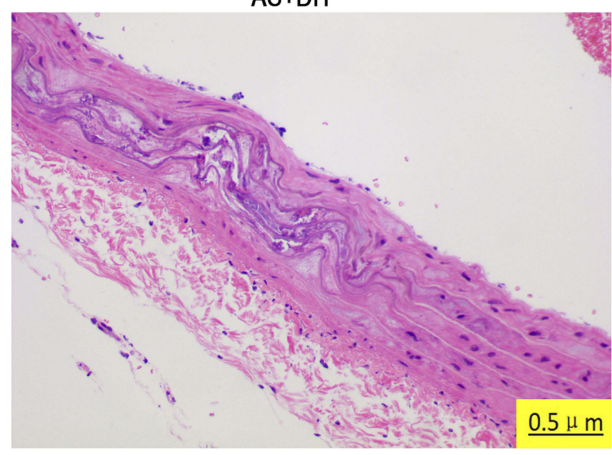

$\mathrm{AS}+\mathrm{DH}+\mathrm{BBR}$

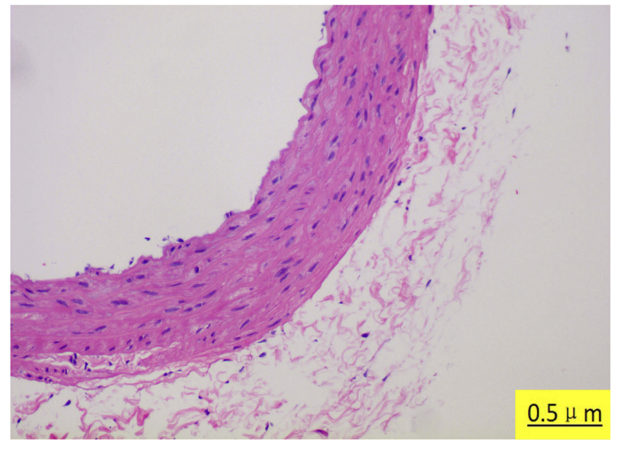

$A S+D H+B B R+3-M A$

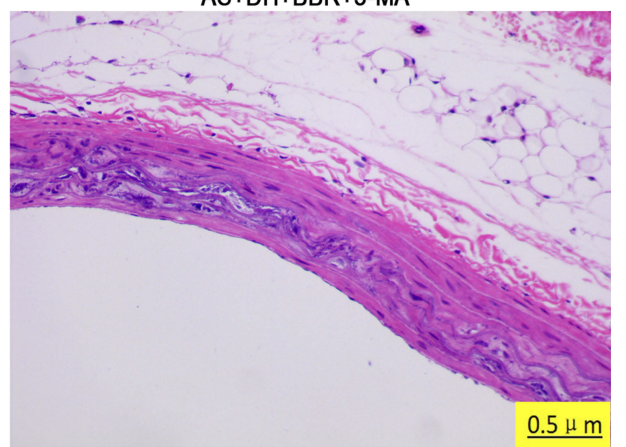

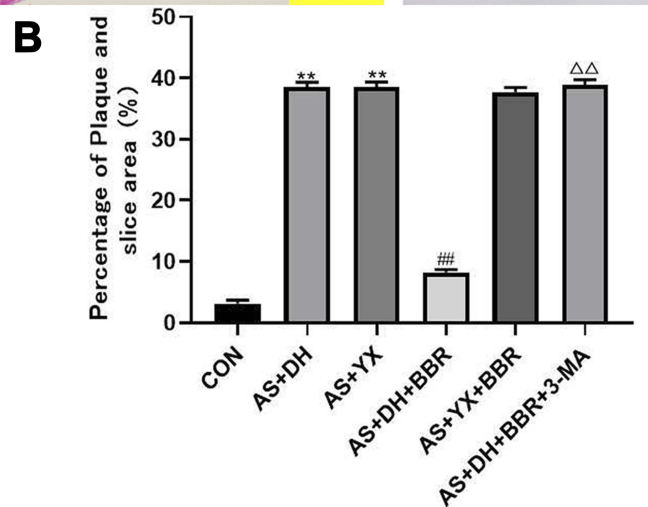

Figure I The histology analysis of the rat aortic tissues. (A) HE staining of the rat aortic tissue sections (200x). (B) Plaque percentage in the section area. Note: The nuclei are blue, and the lipid plaques and foam cells are white. All the experiments were repeated for 5 times. ${ }^{* * P} P<0.01$ vs $C O N$ group; ${ }^{\#} \mathrm{P}<0.01$ vs $A S+D H$ group; ${ }^{\Delta \Delta P}<0.01$ vs $A S+D H+B B R$ group.

LC3-II protein expression level among the CON, AS + DH and $\mathrm{AS}+\mathrm{YX}$ groups $(\mathrm{P}>0.05$; Figure 4$)$, whereas the level of P62 protein expression was significantly increased in AS + $\mathrm{DH}$ and AS + YX group when compared to CON group
$(\mathrm{P}<0.01$; Figure 5). BBR treatment in the AS $+\mathrm{DH}$ rats significantly increased the protein expression level of LC3-II but decreased the protein expression level of P62 when compared to the AS + DH group $(\mathrm{P}<0.01$; Figures 4 and 5$)$. The 


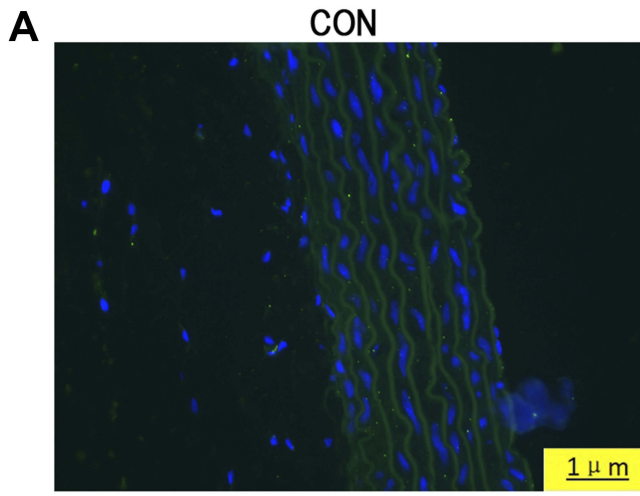

$A S+Y X$

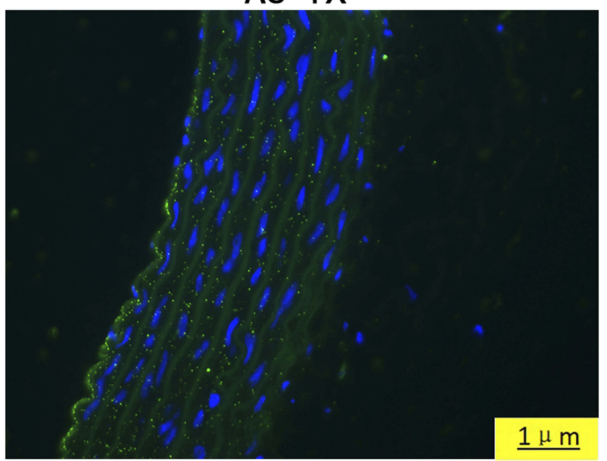

$A S+Y X+B B R$

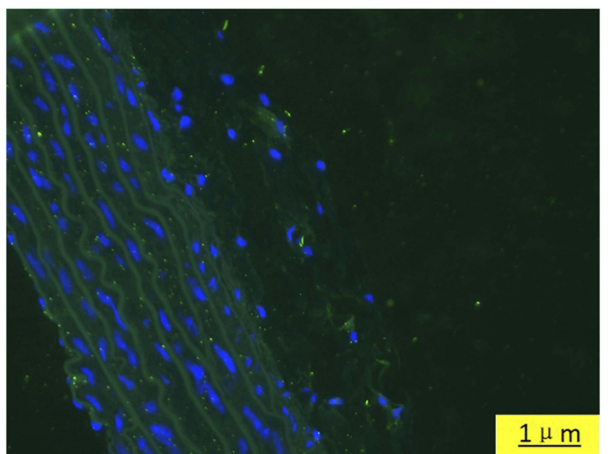

B

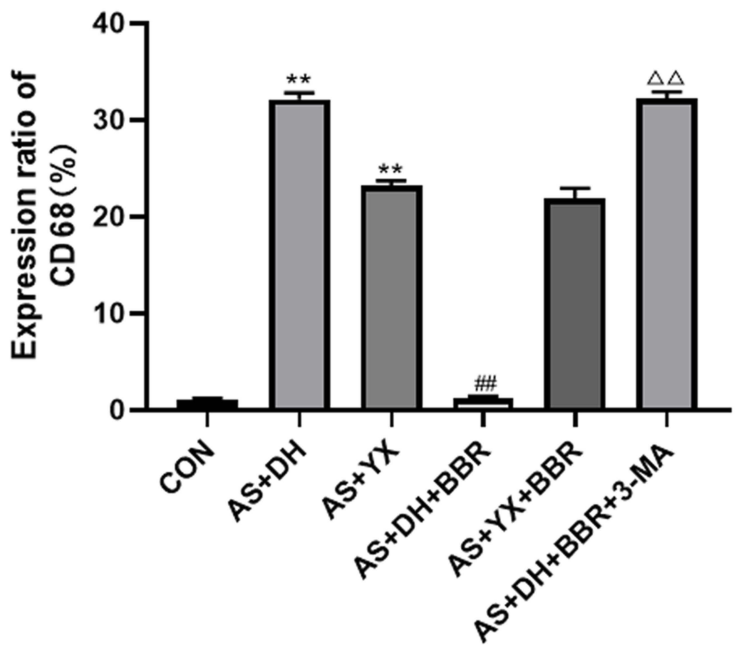

$\mathrm{AS}+\mathrm{DH}$

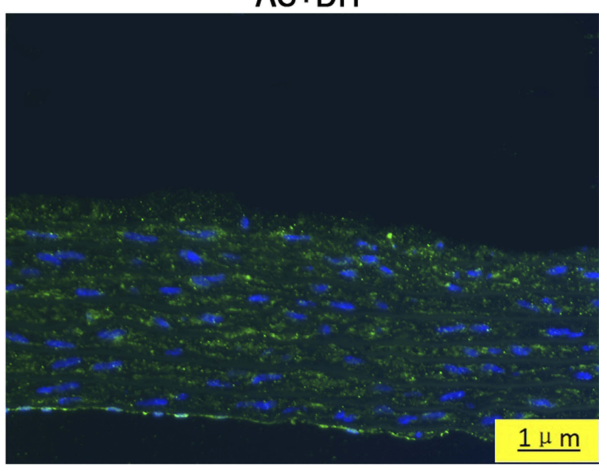

$\mathrm{AS}+\mathrm{DH}+\mathrm{BBR}$

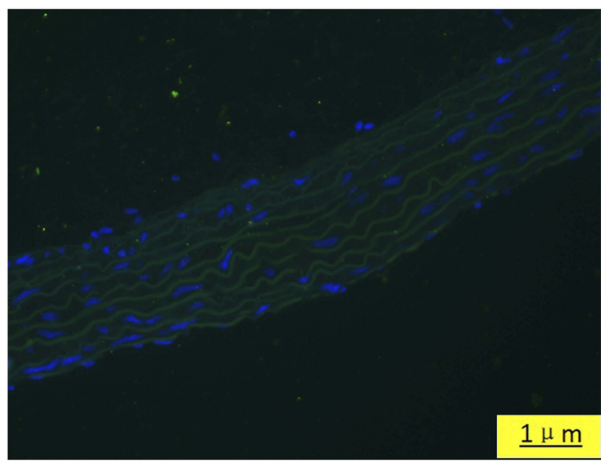

$A S+D H+B B R+3-M A$

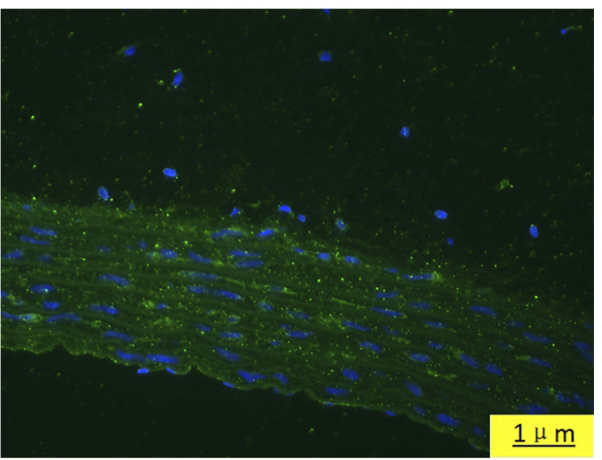

Figure 2 The immunofluorescent analysis of the rat aortic tissues. (A) Immunofluorescence staining of aortic tissue (400x). (B) Rate of CD68 positive expression.

Notes: The blue dots were the nucleus stained by Hoechst, and the green dots indicate the cytoplasm stained with a CD68 fluorescent antibody. All the experiments were repeated for 5 times ${ }^{* *} \mathrm{P}<0.01$ vs $\mathrm{CON}$ group; ${ }^{\# \#} \mathrm{P}<0.01$ vs $\mathrm{AS}+\mathrm{DH}$ group; ${ }^{\Delta \Delta} \mathrm{P}<0.01$ vs $\mathrm{AS}+\mathrm{DH}+\mathrm{BBR}$ group. 

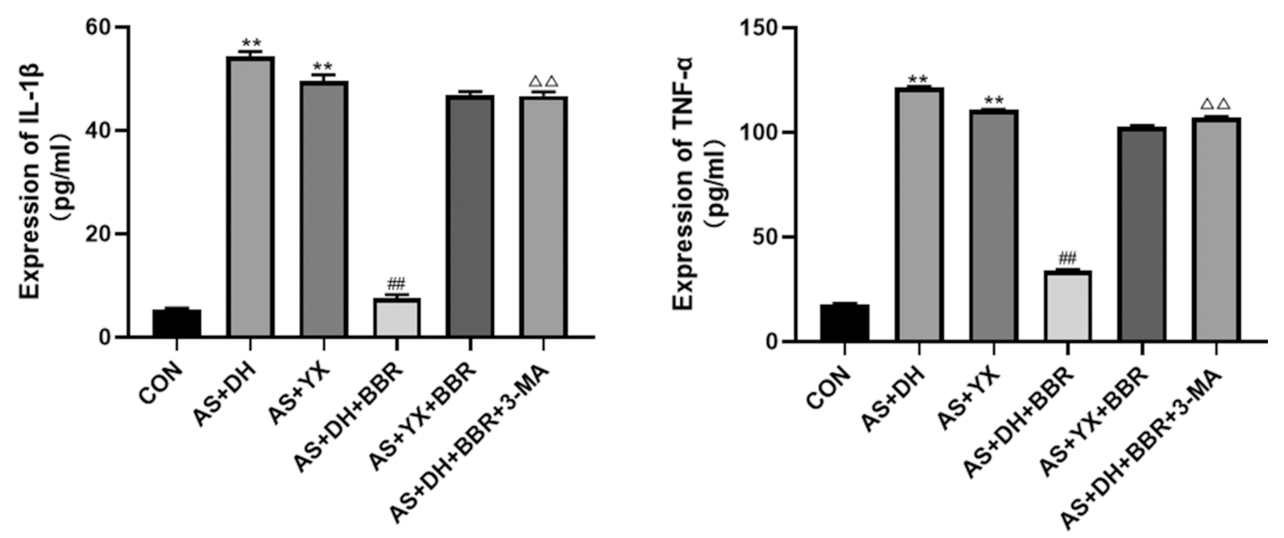

Figure 3 Level of IL-I $\beta$ and TNF- $\alpha$ expression $(n=5)$. All the experiments were repeated for 5 times.

Notes: ${ }^{* * P}<0.01$ vs CON group; ${ }^{\# \#} \mathrm{P}<0.01$ vs $\mathrm{AS}+\mathrm{DH}$ group; ${ }^{\Delta \Delta} \mathrm{P}<0.01$ vs $\mathrm{AS}+\mathrm{DH}+\mathrm{BBR}$ group.

BBR-induced increase in the protein expression level of LC3II and the decreased in the protein expression level of P62 in aortic tissues from the $\mathrm{AS}+\mathrm{DH}$ rats were attenuated by the treatment with 3-MA $(\mathrm{P}<0.01$; Figures 4 and 5). BBR treatment had no significant effects on the LC3-II and P62 protein levels in aortic tissues from $\mathrm{AS}+\mathrm{YX}$ rat $(\mathrm{P}>0.05$; Figures 4 and 5).

\section{Changes of LC3-II and P62 Protein Expression Levels in the Aortic Tissues as Determined by Western Blot}

As shown in Figure 6, Western blot assay was further performed to verify the changes in the expression of LC3-II and P62 in the aortic tissues. There was no significant difference in the LC3-II protein expression level among the CON, AS + $\mathrm{DH}$ and $\mathrm{AS}+\mathrm{YX}$ groups $(\mathrm{P}>0.05$; Figure 6$)$, whereas the protein levels of $\mathrm{P} 62$ protein expression were significantly higher in $\mathrm{AS}+\mathrm{DH}$ and $\mathrm{AS}+\mathrm{YX}$ group than that in the $\mathrm{CON}$ group $(\mathrm{P}<0.01$; Figure 6$)$. BBR treatment in the $\mathrm{AS}+\mathrm{DH}$ rats significantly increased the protein expression level of LC3-II but decreased the protein expression level of P62 when compared to the $\mathrm{AS}+\mathrm{DH}$ group $(\mathrm{P}<0.01$; Figure 6), which was significantly attenuated by 3-MA treatment $(\mathrm{P}<0.01$; Figure 6). BBR treatment had no significant effects on the LC3-II and P62 protein levels in aortic tissues from $\mathrm{AS}+\mathrm{YX}$ rat $(\mathrm{P}>0.05$; Figure 6$)$.

\section{Discussion}

Atherosclerosis (AS) is the pathological basis of various vascular diseases, including coronary atherosclerotic heart disease, ischemic stroke, and lower extremity arterial occlusive disease. Fortunately, the mortality associated with ASinduced diseases has been significantly reduced worldwide.
According to epidemiological data, the mortality rate of AS-related vascular diseases in individuals aged older than 35 years has decreased from $16 \%$ in 1980 to $4 \%$ in $2010 ;{ }^{1}$ however, the incidence of AS in China remains high with one-third of people in China suffering from carotid atherosclerosis. $^{2}$ Thus, further understanding of the pathogenesis of AS and the proposal of more effective prevention and treatment measures are the urgent issues to be solved in clinical practice. Currently, AS is considered to be a chronic inflammatory disease characterized by lipid deposition. ${ }^{25}$ The increase of inflammatory macrophages within plaques leads to excessive activation of an inflammatory reaction, which in turn produces more foam cells, a larger necrotic core area, and a thinner fibrous membrane. ${ }^{26}$ Therefore, an active inflammatory response in the plaque is the main indicator of arterial plaque instability.

It has been suggested that the over-activation of the endoplasmic reticulum during AS formation leads to the overexpression of NF- $\kappa$ B, IL- $1 \beta$, TNF- $\alpha$ and other inflammatory factors, induces complex inflammatory cascades, and participates in the formation of vulnerable plaques. Moreover, under conditions of stress, the endoplasmic reticulum promotes the degradation of damaged mitochondria and inflammation-related proteins by activating autophagy, thereby inhibiting the inflammatory response, further endoplasmic reticulum stress, as well as retarding the progression of plaques. ${ }^{27}$ Fan et al demonstrated that BBR alleviates oxLDL induced inflammatory factors and suggests a protective role for atherosclerosis via induction of autophagy, but Deng et al found out that BBR attenuates autophagy in adipocytes by targeting BECN1. ${ }^{14,28}$ Therefore, the interplay between autophagy and inflammation may represent a key link for the prevention and treatment of AS. 
A

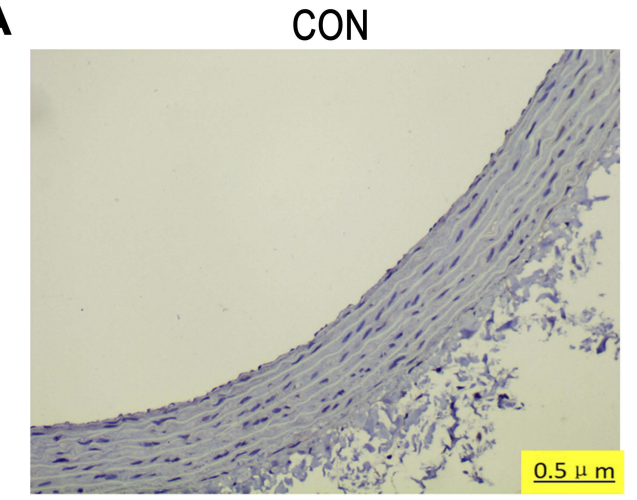

$A S+Y X$
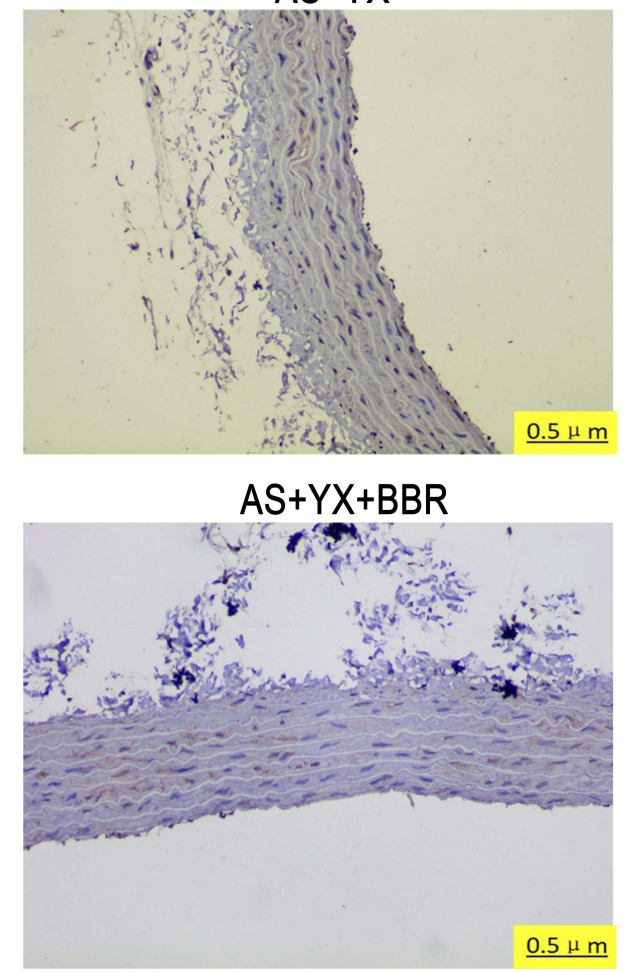

AS+DH

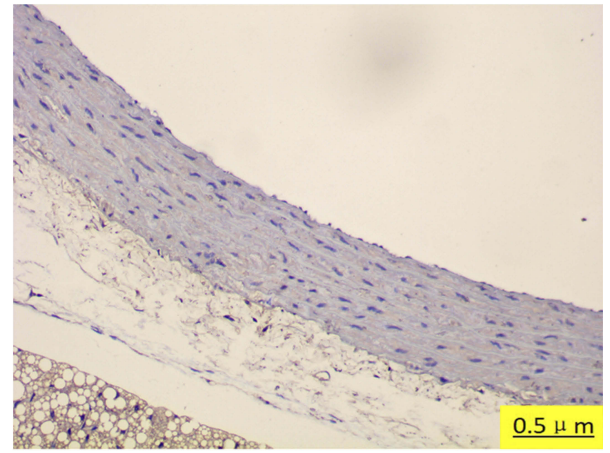

$A S+D H+B B R$
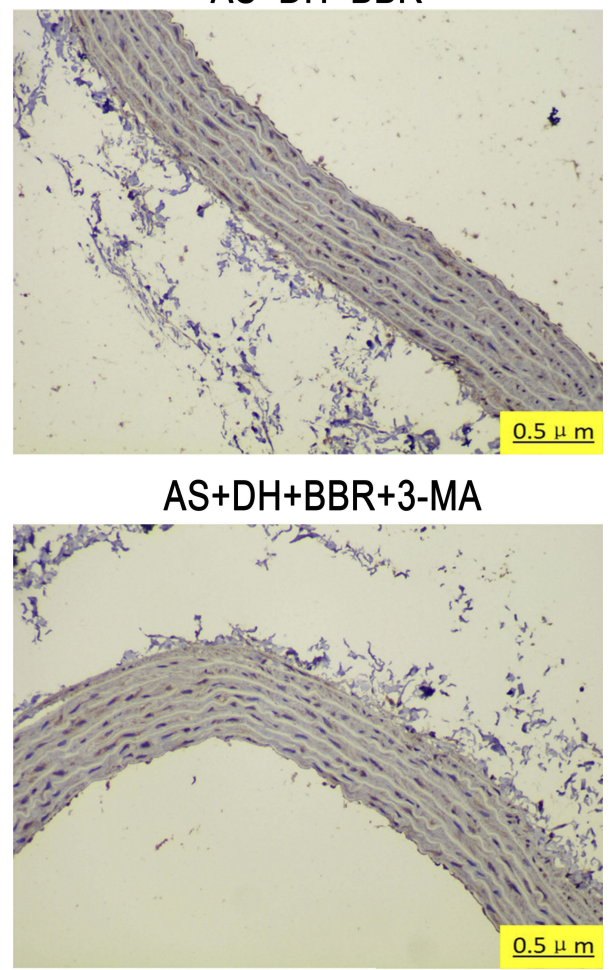

B

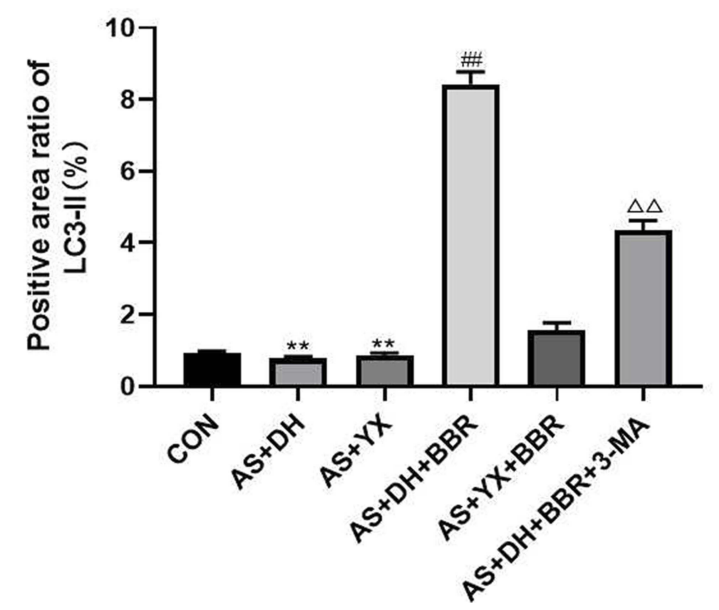

Figure 4 LC3-II protein expression in the rat aortic tissues. (A) Immunohistochemical test LC3-II (200x; $n=5)$. (B) LC3-II protein positive area ratio ( $n=5$ ). Notes: The above blue dot indicates wood staining nuclei, brown dot to dye DAB LC3-II protein. All the experiments were repeated for 5 times. $* * P<0.0$ I vs CON group; ${ }^{\#} \mathrm{P}<0.0 \mathrm{I}$ vs $\mathrm{AS}+\mathrm{DH}$ group; ${ }^{\Delta \Delta} \mathrm{P}<0.0 \mathrm{I}$ vs $\mathrm{AS}+\mathrm{DH}+\mathrm{BBR}$ group. 
A
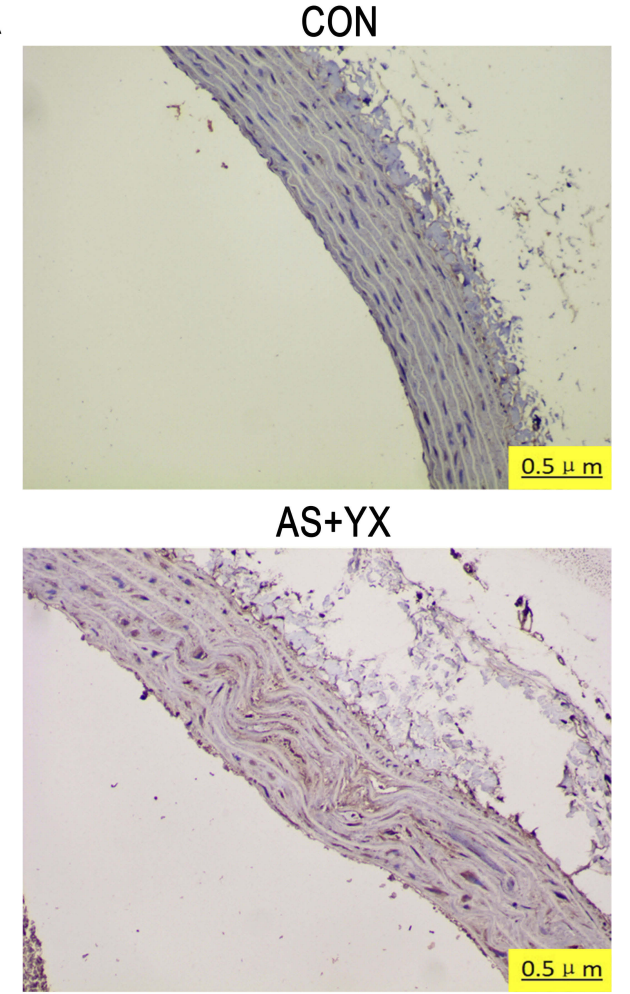

$A S+Y X+B B R$

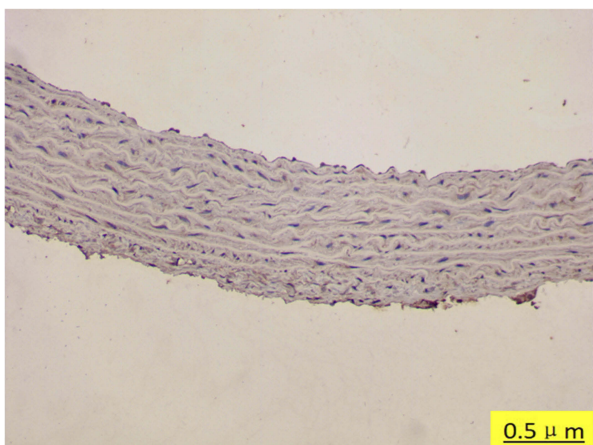

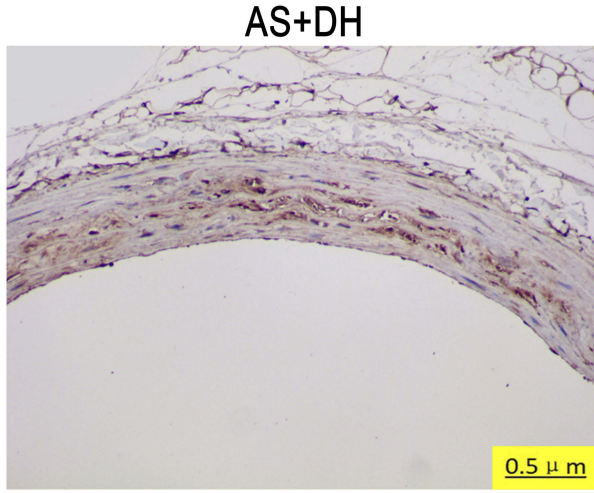

$\mathrm{AS}+\mathrm{DH}+\mathrm{BBR}$

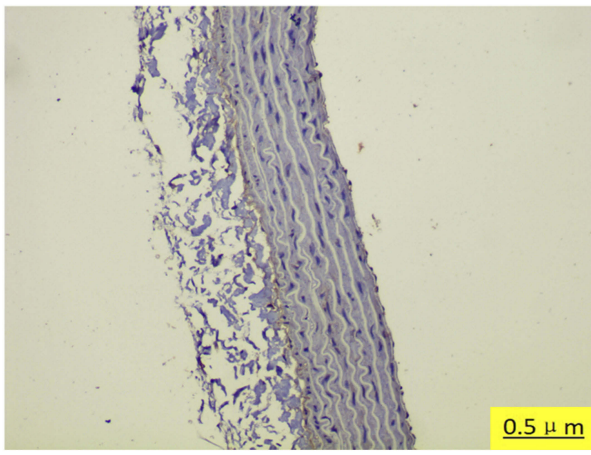

$\mathrm{AS}+\mathrm{DH}+\mathrm{BBR}+3-\mathrm{MA}$

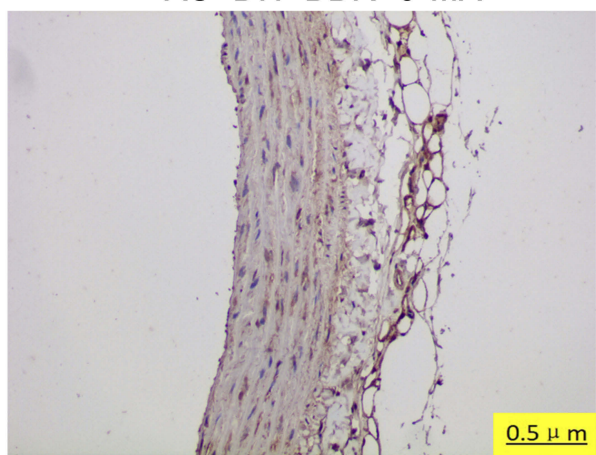

B

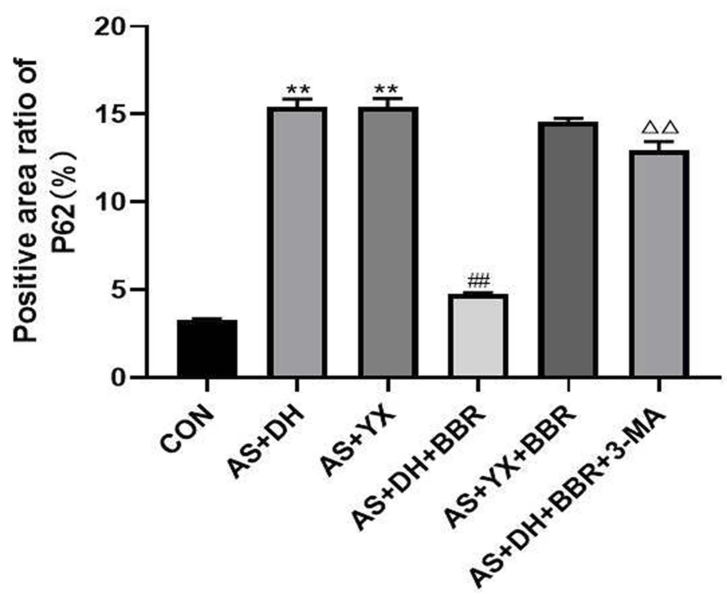

Figure 5 P62 protein expression in the rat aortic tissues. (A) Immunohistochemical detection P62 (200x). (B) P62 protein-positive area ratio.

Notes: The above blue dot indicates wood staining nuclei, brown dot to dye DAB P62 protein. All the experiments were repeated for 5 times. $* * P<0.0$ I vs CON group; ${ }^{\#} \mathrm{P}<0.01$ vs $\mathrm{AS}+\mathrm{DH}$ group; ${ }^{\Delta \Delta} \mathrm{P}<0.01$ vs $\mathrm{AS}+\mathrm{DH}+\mathrm{BBR}$ group. 

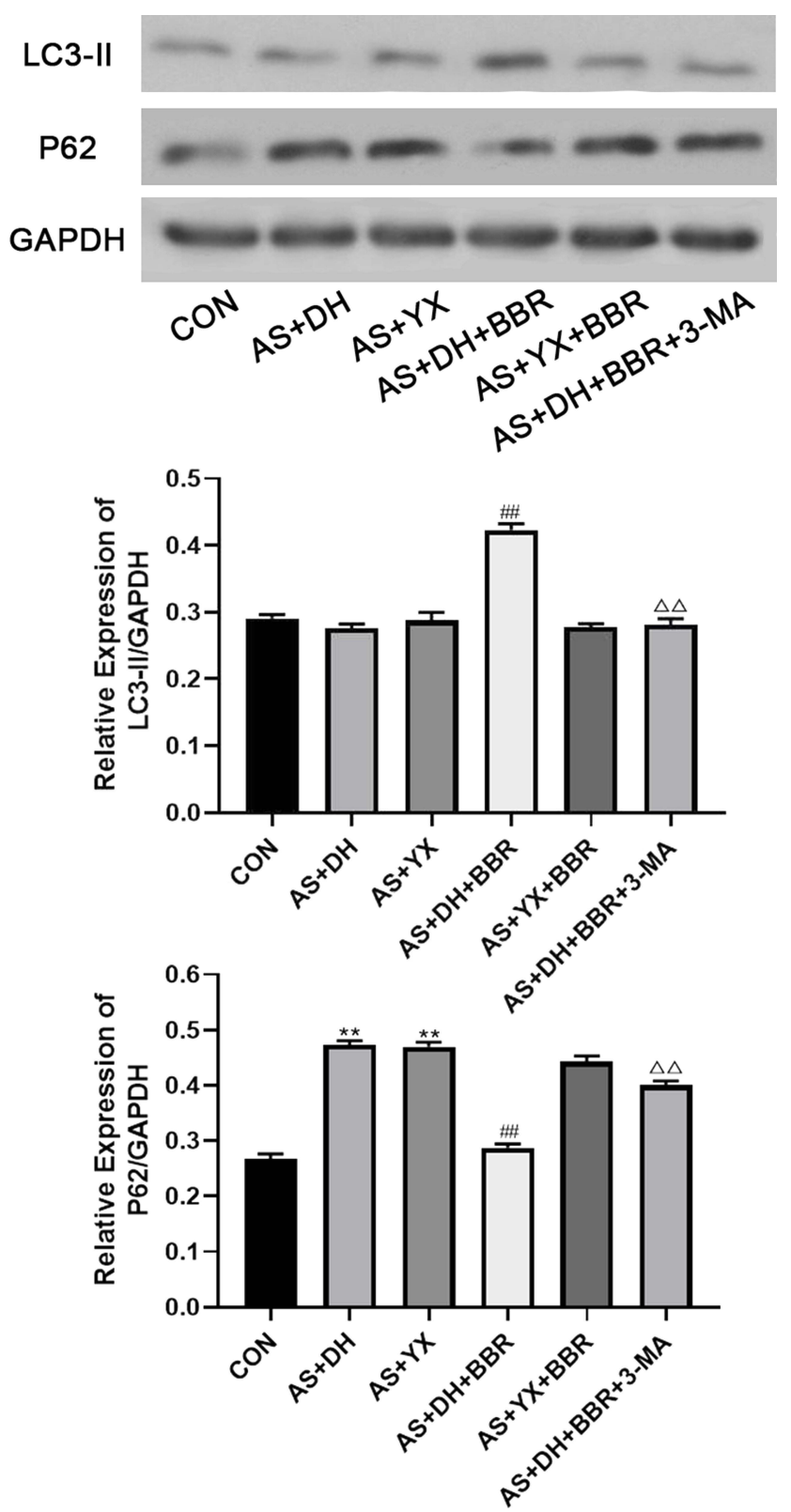

Figure 6 Detection of autophagy marker proteins, LC3-II and P62 by Western blot. All the experiments were repeated for 5 times.

Notes: ${ }^{* * P}<0.01$ vs CON group; ${ }^{\#} \mathrm{P}<0.01$ vs $\mathrm{AS}+\mathrm{DH}$ group; ${ }^{\Delta \Delta} \mathrm{P}<0.01$ vs $\mathrm{AS}+\mathrm{DH}+\mathrm{BBR}$ group.

Traditional Chinese medicine has been shown to be effective for the prevention and treatment of AS. ${ }^{29}$ Some herbal extracts have been shown to reverse the progression of AS by inhibiting the inflammatory responses. For example, ginsenoside F1 significantly increases the activity of endothelial cells injured by ox-LDL, elevated the expression of A20, improves the level of lox-1 and TLR4 expression, and reduced the adhesion of monocytes, thereby inhibiting the inflammatory response and reducing the area of atherosclerotic plaques. ${ }^{30}$
It is important to note that the treatment of diseases by TCM emphasizes the "consistency of disease and syndrome" and "there is the syndrome, there is the medicine". Therefore, establishing the "combination of diseases and syndromes" model and discussing the therapeutic effect of drugs with different syndromes in the same disease will provide more sufficient evidence for the use of TCM to treat AS. As a key traditional Chinese medicine for treating damp-heat syndrome, BBR with a precise anti-inflammatory effect is mainly used to treat acute and chronic inflammatory diseases of the gastrointestinal tract. Currently, pharmacological and clinical studies have confirmed that BBR can inhibit atherosclerotic plaques. ${ }^{31}$ By controlling temperature and humidity, we successfully established an AS model of damp-heat syndrome and yang deficiency syndrome. Our study observed the therapeutic effect of BBR on different AS syndrome types.

We found that there was an abundance of inflammatory factors, including IL- $1 \beta$ and TNF- $\alpha$, detected in the serum of AS rats with characteristics of damp-heat syndrome and yang deficiency syndrome. In addition, a large amount of macrophage infiltration was observed in the pathological sections, indicating that in the case of damp-heat syndrome and yang deficiency syndrome, AS rats have active inflammatory activity and unstable plaques; moreover, the immunohistochemistry and Western blot findings showed that the autophagy marker, LC3-II, was expressed at low levels in the aortic tissue sections of rats, whereas the $\mathrm{P} 62$ protein was highly expressed. Currently, LC3-II is a well-recognized autophagy marker derived from the LC3 protein, When the LC3 protein is formed, its carboxyl terminus will be cleaved by Atg4 to produce LC3-I localized in the cytoplasm. During autophagy, LC3-I is modified and processed by a ubiquitin-like system, including Atg7 and Atg3, then covalently bound to phosphatidylethanolamine to form LC3-II and localize on the autophagosome membrane. Therefore, it is currently believed that the level of LC3-II is positively correlated with that of autophagy. ${ }^{32}$ P62 is a binding protein downstream of LC3 that interacts with LC3 and infiltrates into autophagosomes for degradation. In addition, P62 can be regarded as a degradation substrate for autophagy, and its content is negatively correlated with the level of autophagy. ${ }^{8}$ Therefore, we believe that there is a low autophagy capacity of the cells in AS patients with either damp-heat syndrome or yang deficiency syndrome. Further experiments revealed that BBR could significantly alleviate the infiltration of macrophages in the aortic tissue of AS rats with damp-heat syndrome and yang deficiency, reduce the expression of inflammatory cytokines IL-1 $\beta$ and TNF- $\alpha$ and the formation of foam cells, as well as 
significantly reduce the area of intra-aortic lipid plaques; however, no effect of BBR was observed in AS rats with yang deficiency. This finding indicates that BBR can effectively inhibit AS and increase plaque stability in the state of dampheat syndrome; however, it is ineffective for yang deficiency syndrome AS. This is in contrast to the clinical observation that the heat-clearing and moisture-removing component of Coptis chinensis can effectively improve the clinical symptoms of AS patients with damp-heat syndrome, as well as aggravate the clinical symptoms of AS patients with yang deficiency. This opposing effect can be explained by the TCM theory that the essence of BBR is "cold", and the syndrome of damp-heat is "hot". Thus, when cold treatment is used, heat is dissipated, wet is degenerated, the evil is removed, and the disease is cured. In contrast, since the yang deficiency syndrome is "cold", when it is treated with "cold", the cold elements fight, the evil is increased, and the disease is aggravated. Our further analysis revealed that following BBR intervention, the level of LC3-II expression in the aortic tissue of AS rats with damp-heat syndrome was increased, whereas P62 was significantly decreased. 3-MA is a widely used autophagy inhibitor, which increases the area of aortic plaques in ApoE-/- mice and interdicts the early stage of autophagy. ${ }^{33,34} 3-\mathrm{MA}$ is an autophagy inhibitor and inhibits autophagy by blocking autophagosome formation via the inhibition of type III Phosphatidylinositol 3-kinases (PI-3K). Studies have demonstrated that 3-MA administration attenuated the inhibitory effects of berberine on oxidative stressinduced apoptosis and the enhanced effects of berberine on autophagy in human nucleus pulposus cells. ${ }^{35}$ In our study, the effects of berberine were reversed after an intervention with 3-MA in which the level of LC3-II in the aortic tissue of the rats decreased while the level of P62 was significantly increased. The aforementioned results indicate that BBR can reduce atherosclerosis development of rats with damp-heat syndrome. The associated mechanism may be related to BBRmediated up-regulation of autophagy and inhibition of the inflammatory response in the plaque.

There are several limitations in the current study. Firstly, the underlying molecular mechanisms of the damp-heat syndrome and yang deficiency syndrome have not been fully understood, and we should be careful when interpreting the current findings. Further studies may be required to determine the underlying mechanisms related to these syndromes. Secondly, the present has not compared the effects of berberine on AS rats with other AS rats with damp-heat syndrome and yang deficiency, which may be considered in our future investigation, in order to further understand these animal models. Thirdly, the present study only determined the LC3II and P62 autophagy markers, other markers such as Parkin and Pink1 should be examined to further confirm on the effects of berberine on the autophagy in this specific animal model.

\section{Conclusions}

In conclusion, our studies for the first time examined the effects of BBR on the arterial plaque stability in the AS rats with damp-heat syndrome or yang deficiency syndrome. Our results indicated the BBR could inhibit arterial plaque formation and alleviate the inflammatory response in the aortic tissues in the AS rats with damp-heat syndrome possibly via promoting autophagy. However, the molecular mechanisms of BBR-mediated protective effects in this animal model still require further investigation.

\section{Acknowledgments}

The study was supported by Project funded by China Postdoctoral Science Foundation (NO. 2018M631048 and 2019T120792), the Sanming Project of Medicine in Shenzhen of China (NO.SZSM201612048), the Science and Technology project of Shenzhen city of China (JCYJ20160426100250466 and JCYJ20170306152620264) and the Baoan Science and Technology Innovation Department of Shenzhen City of China (2016CX024 and 2017JD146).

\section{Disclosure}

The authors report no conflicts of interest in this work.

\section{References}

1. Herrington W, Lacey B, Sherliker P, Armitage J, Lewington S Epidemiology of atherosclerosis and the potential to reduce the global burden of atherothrombotic disease. Circ Res. 2016;118(4):535-546. doi:10.1161/CIRCRESAHA.115.307611

2. Clarke R, Du H, Kurmi O, et al. Burden of carotid artery atherosclerosis in Chinese adults: implications for future risk of cardiovascular diseases. Eur J Prev Cardiol. 2017;24(6):647-656. doi:10.1177/ 2047487317689973

3. Galluzzi L, Baehrecke EH, Ballabio A, et al. Molecular definitions of autophagy and related processes. EMBO J. 2017;36(13):1811-1836. doi:10.15252/embj.201796697

4. Netea-Maier RT, Plantinga TS, van de Veerdonk FL, Smit JW, Netea MG. Modulation of inflammation by autophagy: consequences for human disease. Autophagy. 2016;12(2):245-260. doi:10.1080/ 15548627.2015.1071759

5. Xie X, Ma X, Zeng S, et al. Mechanisms of berberine for the treatment of atherosclerosis based on network pharmacology. Evid Based Complement Alternat Med. 2020;2020:3568756. doi:10.1155/2020/ 3568756

6. Zou K, Li Z, Zhang Y, et al. Advances in the study of berberine and its derivatives: a focus on anti-inflammatory and anti-tumor effects in the digestive system. Acta Pharmacol Sin. 2017;38(2):157-167. doi:10.1038/aps.2016.125 
7. Liu CS, Zheng YR, Zhang YF, Long XY. Research progress on berberine with a special focus on its oral bioavailability. Fitoterapia. 2016;109:274-282. doi:10.1016/j.fitote.2016.02.001

8. Song P, Li S, Wu H, et al. Parkin promotes proteasomal degradation of p62: implication of selective vulnerability of neuronal cells in the pathogenesis of Parkinson's disease. Protein Cell. 2016;7 (2):114-129. doi:10.1007/s13238-015-0230-9

9. Zhu L, Zhang D, Zhu H, et al. Berberine treatment increases akkermansia in the gut and improves high-fat diet-induced atherosclerosis in Apoe(-/-) mice. Atherosclerosis. 2018;268:117-126. doi:10.1016/j. atherosclerosis.2017.11.023

10. Wu M, Yang S, Wang S, et al. Effect of berberine on atherosclerosis and gut microbiota modulation and their correlation in high-fat diet-fed ApoE-/- mice. Front Pharmacol. 2020;11:223.

11. Mohammadinejad R, Ahmadi Z, Tavakol S, Ashrafizadeh M. Berberine as a potential autophagy modulator. J Cell Physiol. 2019;234(9):14914-14926. doi:10.1002/jcp.28325

12. Huang Z, Han Z, Ye B, et al. Berberine alleviates cardiac ischemia/ reperfusion injury by inhibiting excessive autophagy in cardiomyocytes. Eur J Pharmacol. 2015;762:1-10. doi:10.1016/j.ejphar.2015.05.028

13. $\mathrm{Li} \mathrm{MH}$, Zhang $\mathrm{YJ}, \mathrm{Yu} \mathrm{YH}$, et al. Berberine improves pressure overload-induced cardiac hypertrophy and dysfunction through enhanced autophagy. Eur J Pharmacol. 2014;728:67-76. doi:10.1016/ j.ejphar.2014.01.061

14. Fan X, Wang J, Hou J, et al. Berberine alleviates ox-LDL induced inflammatory factors by up-regulation of autophagy via AMPK/ mTOR signaling pathway. J Transl Med. 2015;13(1):92. doi:10. 1186/s12967-015-0450-z

15. Lin WX, Du X, Yang LL, et al. Differences in the composition of vaginal microbiota between women exhibiting spleen-deficiency syndrome and women with damp-heat syndrome, two of the most common syndromes of vaginitis in traditional chinese medicine. Evid Based Complement Alternat Med. 2019;2019:5456379. doi:10.1155/2019/5456379

16. Zhang SJ, Chen Z, Li GW, Wang BL. Effect of the haoqinqingdan decoction on damp-heat syndrome in rats with influenza viral pneumonia. Asian Pac J Trop Med. 2013;6(8):653-657. doi:10.1016/S1995-7645(13)60113-3

17. Shen J, Chen JJ, Zhang BM, et al. Baicalin is curative against rotavirus damp heat diarrhea by tuning colonic mucosal barrier and lung immune function. Dig Dis Sci. 2019. doi:10.1007/s10620-019-05977-w

18. Yao C, Wang L, Zhang J, Zhou X, Zhang C. Immunotoxicity of hydrocortisone on Th1/Th2-related cytokine production is associated with Yang-deficient state in traditional Chinese medicine. Cell Mol Immunol. 2007;4(5):383-388.

19. Xiong R, Li Y, Zheng K, et al. Er Shen Wan extract alleviates polyuria and regulates AQP 2 and AVPR 2 in a rat model of spleen-kidney Yang deficiency-induced diarrhea. Biomed Pharmacother. 2019;110:302-311. doi:10.1016/j.biopha.2018.11.147

20. Du K, Gao XX, Feng Y, et al. Integrated adrenal and testicular metabolomics revealed the protective effects of Guilingji on the kidney-Yang deficiency syndrome rats. $J$ Ethnopharmacol. 2020;255:112734. doi:10.1016/j.jep.2020.112734
21. Xiong R, Li W, Li Y, et al. Er Shen Wan extract reduces diarrhea and regulates AQP 4 and NHE 3 in a rat model of spleen-kidney Yang deficiency-induced diarrhea. Biomed Pharmacother. 2018;98:834-846. doi:10.1016/j.biopha.2018.01.023

22. Ren X, Wen X, Hong B, Liao Y, Ma W, Tang Y. Effects of sanren decoction on Th1/Th2 cytokines in rats with spleen-stomach damp-heat syndrome. Nan Fang Yi Ke Da Xue Xue Bao. 2012;32(2):181-184.

23. Chen R, Wang J, Zhan R, Zhang L, Wang X. Fecal metabonomics combined with $16 \mathrm{~S}$ rRNA gene sequencing to analyze the changes of gut microbiota in rats with kidney-yang deficiency syndrome and the intervention effect of You-gui pill. J Ethnopharmacol. 2019;244: 112139. doi:10.1016/j.jep.2019.112139

24. Singh N, Sharma B. Toxicological effects of berberine and sanguinarine. Front Mol Biosci. 2018;5:21. doi:10.3389/fmolb.2018.00021

25. Geovanini GR, Libby P. Atherosclerosis and inflammation: overview and updates. Clin Sci. 2018;132(12):1243-1252. doi:10.1042/CS20180306

26. Kasikara C, Doran AC, Cai B, Tabas I. The role of non-resolving inflammation in atherosclerosis. $J$ Clin Invest. 2018;128 (7):2713-2723. doi:10.1172/JCI97950

27. Zhang C, Syed TW, Liu R, Yu J. Role of endoplasmic reticulum stress, autophagy, and inflammation in cardiovascular disease. Front Cardiovasc Med. 2017;4:29. doi:10.3389/fcvm.2017.00029

28. Deng Y, Xu J, Zhang X, et al. Berberine attenuates autophagy in adipocytes by targeting BECN1. Autophagy. 2014;10(10):1776-1786. doi:10.4161/auto.29746

29. Wang C, Niimi M, Watanabe T, Wang Y, Liang J, Fan J. Treatment of atherosclerosis by traditional Chinese medicine: questions and quandaries. Atherosclerosis. 2018;277:136-144. doi:10.1016/j. atherosclerosis.2018.08.039

30. Qin M, Luo Y, Lu S, et al. Ginsenoside F1 ameliorates endothelial cell inflammatory injury and prevents atherosclerosis in mice through A20-mediated suppression of NF-kB signaling. Front Pharmacol. 2017;8:953. doi:10.3389/fphar.2017.00953

31. Wan Q, Liu Z, Yang Y, Cui X. Suppressive effects of berberine on atherosclerosis via downregulating visfatin expression and attenuating visfatin-induced endothelial dysfunction. Int J Mol Med. 2018;41 (4):1939-1948. doi:10.3892/ijmm.2018.3440

32. Xu Y, Zhang J, Tian C, et al. Overexpression of p62/SQSTM1 promotes the degradations of abnormally accumulated PrP mutants in cytoplasm and relieves the associated cytotoxicities via autophagy-lysosome-dependent way. Med Microbiol Immunol. 2014;203(2):73-84. doi:10.1007/s00430-013-0316-z

33. Klionsky DJ, Abdalla FC, Abeliovich H, et al. Guidelines for the use and interpretation of assays for monitoring autophagy. Autophagy. 2012;8(4):445-544.

34. Cao H, Jia Q, Shen D, Yan L, Chen C, Xing S. Quercetin has a protective effect on atherosclerosis via enhancement of autophagy in ApoE(-/-) mice. Exp Ther Med. 2019;18(4):2451-2458. doi:10.3892/etm.2019.7851

35. Luo R, Liao Z, Song $Y$, et al. Berberine ameliorates oxidative stress-induced apoptosis by modulating ER stress and autophagy in human nucleus pulposus cells. Life Sci. 2019;228:85-97. doi:10.1016/j.1fs.2019.04.064

\section{Publish your work in this journal}

Drug Design, Development and Therapy is an international, peerreviewed open-access journal that spans the spectrum of drug design and development through to clinical applications. Clinical outcomes, patient safety, and programs for the development and effective, safe, and sustained use of medicines are a feature of the journal, which has also been accepted for indexing on PubMed Central. The manuscript management system is completely online and includes a very quick and fair peer-review system, which is all easy to use. Visit http://www. dovepress.com/testimonials.php to read real quotes from published authors. 